\title{
The Use of Magnetocardiography and Body Surface Potential Mapping in the Detection of Coronary Artery Disease in Chest Pain Patients with a Normal Electrocardiogram
}

\author{
R Hoekema ${ }^{1}$, C Gürlek $^{1}$, MA Brouwer $^{1}$, I Chaikovsky $^{2}$, FWA Verheugt ${ }^{1}$ \\ ${ }^{1}$ Heartcenter, Cardiology Dept., University Medical Center St Radboud Nijmegen, The Netherlands \\ ${ }^{2}$ MagScan GmbH \& Co.KG, Essen Germany
}

\begin{abstract}
In this study, we have tested both the 36-channel Magnetocardiogram (MCG) and the 40-lead Body Surface Potential Map (BSPM) in their performance of diagnosing significant coronary artery disease in 27 chest pain patients with a normal electrocardiogram (ECG) and 23 healhty controls.

The analysis of the BSPMs and MCGs was focused on several aspects of the ST segment.Using discriminant analysis on parameters describing the morphology of the $M C G$ and BSPM tracings, it was found that for classification in three groups, (healthy subjects, and chest pain patients with and without significant coronary artery disease (CAD)), at most a correct classification ratio of $74 \%, 74 \%$ and $76 \%$ can be obtained for respectively $M C G, B S P M$ and $M C G / B S P M$ combined.

It was shown that with $M C G$ and BSPM provide additional, noninvasive, regional information compared to 12-lead resting ECG, which could help in the discrimination of chest pain patients with and without significant $C A D$.
\end{abstract}

\section{Introduction}

In a subgroup of patients with chest pain, suggestive of angina pectoris (AP), it is difficult to determine the cause of the symptoms because the commonly used diagnostic techniques, such as the resting electrocardiogram (ECG), do not reveal any abnormalities. In those patients, additional diagnostic procedures are necessary, such as the rather insensitive bicycle test or the time consuming, expensive and invasive coronary angiogram (CAG). If it were possible to diagnose these patients rapidly and with low costs, it would be of benefit in determining further treatment of these patients. One such test could be the magnetocardiogram (MCG). With this technique, the magnetic field associated with the electrical activity of the heart is measured. No contact with the patient is made and measurement of the MCG is relatively inexpensive, non-invasive and time efficient. However, its sensitivity and specificity are only tested in a few small scaled studies.

The magnetical and electrical field of a certain electrical source are directly correlated with each other (see e.g.[1]). Hence, the question arises what the additional value of MCG is if we compare it with an adequate measurement of the electrical field of the same source, such as a Body Surface Potential Map (BSPM), which is an electrocardiogram, measured by using a large number of electrodes, placed at many positions on the thorax. In this study, we have tested both the MCG and the BSPM in their performance of diagnosing significant coronary artery disease in chest pain patients.

\section{Methods}

\subsection{Patient selection}

Three groups of individuals were recruited for this study:

Group 1. Healthy volunteers, whose 12-lead resting ECG showed no abnormalities.

Group 2. Patients with angina pectoris and a normal resting 12-lead ECG. Their coronary angiogram showed no significant stenoses. The left ventriculogram of these patients showed no abnormalities.

Group 3. Patients with angina pectoris, a normal resting 12-lead ECG, and a significant ( $>50 \%)$ stenosis in one or more coronary arteries, proven by angiography. The left ventriculogram of these patients showed no abnormalities.

The patients were selected from a database of 800 coronary angiography reports and their latest resting 12lead ECG as present in the patient file. Patients who underwent PTCA or CABG between CAG and time of selection were excluded. The healthy subjects were mainly employees of the Cardiology department or relatives of those.

\subsection{MCG and BSPM measurements}

BSPM and MCG data were obtained sequentially from all subjects. The MCG measurements were performed in 
a truck trailer that was on the hospital grounds. In the truck, a 4-channel MCG measuring device was placed, that was placed at 9 different locations above the thorax of a subject in supine position. Thus, a signal-averaged 36-lead MCG was measured (6 x 6 array).

Within one hour after the MCG measurement, the BSPM was measured. Eight strips of five electrodes were positioned on the torso of the subject. Five strips were placed vertically on the left side of the thorax and three on the right side. The electrode locations of the precordial leads (V1-V6, V3R and V4R) were a subset of the electrode array. Furthermore, a reference electrode was placed on each limb. Six 10-second recordings were made[2], while the patient was in supine position. Figure 1 shows the electrode set-up. From the BSPMs, 12-lead ECGs were derived. Abnormalities in these ECG's (determined by an expert cardiologist) led to exclusion of the subject in the subsequent analysis.

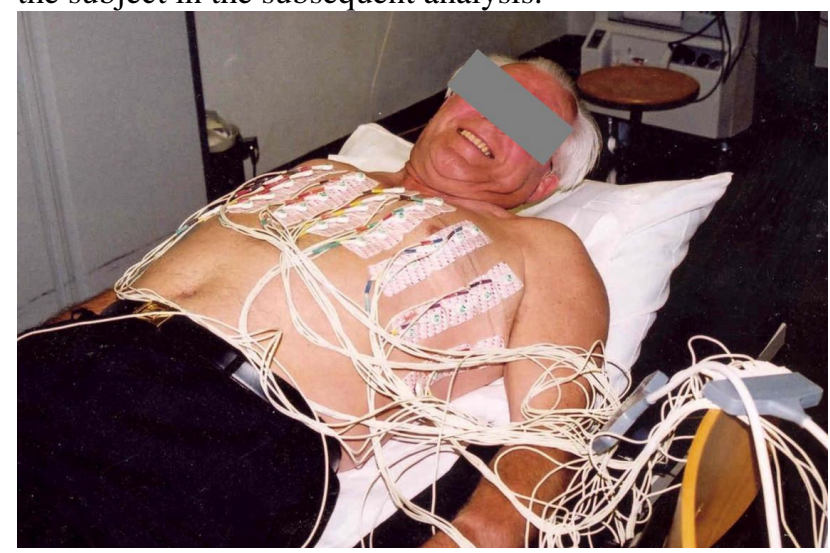

Figure 1. Patient with BSPM electrodes attached.

\subsection{Waveform analysis}

In patients with angina pectoris, it is expected that there are changes in the repolarization of the cardiac cells. Hence, any abnormalities in the ECG and MCG, should come to expression in the ST segment of the cardiac cycle. Therefore, the subsequent analysis was focused on several aspects of the ST segment. It was chosen to use easily derivable features for classifying the data, allowing for easy implementation into clinical practice when proven useful.

The MCG system was capable of automatically creating Current Density Maps from the MCGs, using a semi-infinite medium approach. This rather crude assumption of the geometry of the subjects quickly yields an approximation of the current distribution near the heart, which may be adequate enough for classifying the data. Therefore, classification based on these maps was also studied.

More advanced methods, including the use of sophisticated inverse techniques to determine the electrical heart activity, require accurate knowledge of the geometry of the patient (for example determined by MRI) and user interaction, which makes those methods more difficult to implement into clinical practice.

\subsection{Classification based on Morphology of MCG and BSPM signal}

A method for studying repolarization aspects of the MCG signal and BSPM signal as function of time was described by Hänninen et. al[3,4], where the integral of the measured amplitudes of the second quarter $(1 / 4-1 / 2)$ of the ST-interval was computed and compared between patients and healthy subjects before and after a bicycle test performed during MCG registration. We computed the same integral for each lead. Furthermore, we determined the up-and downslope of the T-Top, as well as timing differences of the maximum of the T-top in individual leads.

A powerful tool to determine whether any classification of data is possible, is discriminant analysis. This technique (part of the statistical analysis software package SPSS) determines from a large set of data, that subset of variables and their respective cut-off values which will lead to a correct classification of the subjects in the patient groups. Prior knowledge (patient group membership) is used to determine which data discriminate best between different patient groups. It will only give an upper limit of the achievable classification rate. We used discriminant analysis to determine whether classification is at all possible, given the morphological ST-segment related data. Data used in the analysis were the group number of each subject and for each channel (lead) in each subject, the integral amplitude of $(1 / 4-1 / 2)$ part of the ST segment, the amplitude and (relative) timing of the T-top, and upward and downward slopes of the T-top.

\subsection{Classification based on Current Density Maps}

The Current Density Maps as described in 2.3 were classified by an experienced, independent observer into

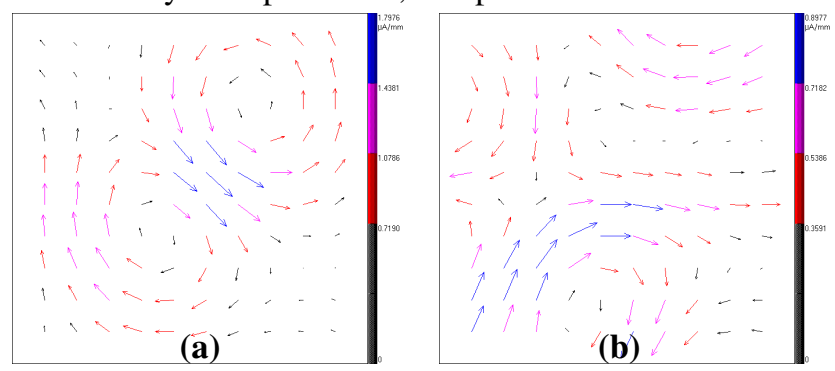

Figure 2. Current density map of a healthy volunteer (a) and a CAD patient with 1-vessel disease (b), both with a normal ECG. 
two groups: "healthy" or "not healthy", based on the pattern visible in these CDMs. A normal pattern consists of two equally sized areas of opposite polarity and a cardiac current vector directed downwards and to the left (Figure 2a). The main feature of pathological pattern is a presence of non-dipole structure (see Figure 2b). The classification was compared with the group membership of each individual.

\section{Results}

\subsection{Patient selection and MCG and BSPM measurements}

Measurements were made on 56 individuals. Four individuals were excluded from the study because of implantation of a metallic intracoronary stent between CAG and MCG/BSPM measurement and two persons had an electrocardiographic reason for their exclusion (LBBB, dextrocardia). Of the remaining 50 subjects, both MCG and BSPM were available in 42 subjects. The MCG was available in 4 more subjects, and BSPM was recorded in 4 other individuals (see Table 1 for details). The time between CAG and the MCG and BSPM measurements ranged from 69 days to 3 years (average 501 days).

Table 1. Demographic data of subjects included in this study. The total number of subjects (No. subjects) in each group is given, as well as the number of males (in parenthesis)

\begin{tabular}{lcccc}
\hline Group & 1 & 2 & 3 & Total \\
\hline No.subjects & $23(15)$ & $10(5)$ & $17(13)$ & $50(33)$ \\
BSPM only & $19(11)$ & $10(5)$ & $17(13)$ & $46(29)$ \\
MCG only & $23(15)$ & $8(4)$ & $15(13)$ & $46(32)$ \\
MCG and BSPM & $19(11)$ & $8(4)$ & $15(13)$ & $42(28)$ \\
Age (years) & 52 & 58 & 61 & 56 \\
Weight $(\mathrm{kg})$ & 77 & 81 & 80 & 79 \\
Length $(\mathrm{cm})$ & 175 & 173 & 173 & 174 \\
\hline
\end{tabular}

\subsection{Classification based on Morphology of MCG and BSPM signal}

The correct classification ratios obtained with the discriminant analysis, using the integral of the $(1 / 4-1 / 2)$ part of the ST-interval, are given in Table 2. We determined these correct classification rates for three groups, as well as for group 2 and 3 pooled together ("healthy" vs

Table 2. Correct classification rates for BSPM, MCG and MCG/BSPM combined using discriminant analysis.

\begin{tabular}{lcc}
\hline Groups & $1,2,3$ & healthy vs. not healthy \\
\hline BSPM & $74 \%$ & $76 \%$ \\
MCG & $74 \%$ & $88 \%$ \\
MCG+BSPM & $76 \%$ & $90 \%$ \\
\hline
\end{tabular}

"not healthy"). When other morphological data (e.g. Ttop slopes) were used, discriminant analysis showed worse correct classification ratios.

\subsection{Classification based on Current Density Maps}

The current density maps, as computed from the MCG data, were classified into two groups: "healthy" and "not healthy", based on the patterns visible in the CDM's. The classification is shown in Table 3 . When pooling the patient groups 2 and 3, a correct classification ratio of $80 \%$ was obtained.

Table 3. Classification based on current density maps.

\begin{tabular}{lcccc}
\hline Group & 1 & 2 & 3 & Total \\
\hline Healthy & 2 & 5 & 11 & 18 \\
Not healty & 21 & 3 & 4 & 28 \\
Total & 23 & 8 & 15 & 46 \\
\hline
\end{tabular}

\section{Discussion and conclusions}

In this research, we have studied the use of BSPM and MCG in the classification of patients with angina pectoris and a significant coronary artery stenosis, of patients with angina pectoris without significant signs of coronary artery disease, and of normal subjects. The BSPM and MCG were measured when the subjects were in supine position and at rest. It was chosen to focus on the ST segment of the cardiac cycle, because the electrical behavior of cardiac cells of patients with significant coronary artery stenosis is assumed to be different from healthy subjects during the repolarization period. Several features of the ST segment were studied.

With discriminant analysis on the morphological data (integral of the $(1 / 4-1 / 2)$ part of the ST-segment for separate channels/leads), it was found that at most $76 \%$ of the patients could be classified correctly, and $90 \%$ if the two patient groups were pooled together. Prior knowledge of the patient's state of health was used, which makes this method only suitable for determining an upper limit of the correct classification ratio. The method automatically determines which data and what threshold is used for a proper classification, without any physiological knowledge involved. Hence, the method tells us that some mathematical differences do exist. Further analysis of the selected channels (leads) in the discriminant analysis may reveal the location of regional differences in the MCG and BSPM data.

In the majority of patients the time between CAG and our measurements was rather long. As a consequence, current coronary status may have changed from that defined as criterion for inclusion of patients in group 2 or 3 . Therefore, pooling groups 2 and 3 in one group is physiologically reasonable. 
The CDM method, in which only a classification into two groups was made, led to a correct classification ratio of $80 \%$. With the CDM method a current density distribution on a plane through the heart is computed by assuming an semi-infinite homogeneous medium. This may be useful in many applications for classifying patients with cardiac disease, although the resulting current density is only an approximation of the electrical heart activity of those subjects. The classification results in this study were promising for two groups but classification will have to be extended to three groups.

The results indicate that with the BSPM and the MCG, additional information about the state of health is obtained when compared to the standard 12-lead resting ECG alone, since the standard 12-lead ECG was classified as normal for all subjects in this study. With BSPM/MCG combined, a still better classification is possible. The classification results for BSPM and MCG are comparable. Clinical application of these methods still has to be discussed. The classification ratio is not much higher than that obtained from exercise tests in routine use, but some disadvantages and complications of a stress test are avoided in these techniques, where measurements are conducted at rest.

Further study may yield better classification methods for these patient groups. For example, more advanced inverse techniques to determine the electrical heart activity could be used to obtain more detailed information about the process of depolarization and repolarization at the heart's surface. These data would probably give more insight in the coronary status of these subjects and hence improve the classification results. Drawback of these more advanced techniques is that they require realistic geometrical data from each subject[5], for example obtained by MR or CT, as well as a considerable amount of user interaction and computing time. As geometrical data of the subjects were not available in this study, these inverse solutions have not been applied.

In this study, it was chosen to measure the resting BSPM and MCG, although it is reported in literature $[3,4,6]$ that classification results improve dramatically when using exercise tests. However, measurement time increases when using exercise tests instead of measurements at rest. Since the BSPM and MCG could not be measured simultaneously, this study would have required that all subjects performed the exercise test twice, at equal level of output, which was considered not to be reproducible.

The question remains whether a classification based on MCG is superior to a classification based on BSPM. In our results, only marginal differences were found between the two types of data. As expected, the maximal classification results were achieved when BSPM and MCG data were combined. In case adequate classification methods might ensue, the choice in favor of BSPM or MCG will probably have to be made based on secondary issues, such as the recording costs, the time efficiency and the availability of data analysis techniques, such as the CDM method.

In conclusion, this study has shown that with MCG and BSPM additional, noninvasive, regional information is available, as compared to the standard 12-lead resting ECG, which could help in the discrimination of chest pain patients with and without significant coronary artery disease. Further study on classification methods and the amount of patient activity during measurement is necessary to prove the clinical usefulness of MCG and BSPM in these patient groups.

A more detailed paper on this study will appear in [7].

\section{Acknowledgements}

We would like to thank SQUID A.G. for making available the MCG system and Mr. B. Awolin and Mr. I. Nedayvoda for their great help in measuring the MCGs and interpreting the CDM's, Dr. A.C. Linnenbank is thanked for making available the BSPM system.

\section{References}

[1] Malmivuo J. and Plonsey R., Bioelectromagnetism, New York, Oxford University Press, 1996

[2] Metting van Rijn AC, Kuiper AP, Linnenbank AC, Grimbergen CA. Patient isolation in multichannel bioelectric recordings by digital transmission through a single optical fiber. IEEE Trans Biomed Engr. 1993; 40:302-8

[3] Hanninen H, Takala P, Korhonen P, Oikarinen L, Makijarvi M, Nenonen J, Katila T, Toivonen L. Features of ST segment and T-wave in exercise-induced myocardial ischemia evaluated with multichannel magnetocardiography. Ann Med. 2002; 34:120-9.

[4] Hanninen H, Takala P, Makijarvi M, Montonen J, Korhonen P, Oikarinen L, Simelius K, Nenonen J, Katila $\mathrm{T}$, Toivonen L. Recording locations in multichannel magnetocardiography and body surface potential mapping sensitive for regional exercise-induced myocardial ischemia. Basic Res Cardiol. 2001; 96:405-14.

[5] van Oosterom A, Huiskamp GJ., A realistic torso model for magnetocardiography. Int J Card Imaging. 1991; 7:169-76.

[6] Kanzaki H, Nakatani S, Kandori A, Tsukada K, Miyatake $\mathrm{K}$. A new screening method to diagnose coronary artery disease using multichannel magnetocardiogram and simple exercise. Basic Res Cardiol. 2003; 98:124-32.

[7] Recent advances in MCG, P. Hugenholtz ed., in press.

Address for correspondence

Rudi Hoekema,

540 Deparment of Cardiology,

University Medical Center Nijmegen,

PO box 9101

6500 HB Nijmegen the Netherlands

e-mail: r.hoekema@cardio.umcn.nl 